\title{
The Impact of a Faculty Development Program, the Leadership in Academic Medicine Program (LAMP), On Self-Efficacy, Academic Promotion and Institutional Retention
}

Judy Tung ( $\sim$ jut9005@med.cornell.edu )

Weill Cornell Medicine

Musarrat Nahid

Weill Cornell Medicine

Mangala Rajan

Weill Cornell Medicine

Lia Logio

Case Western Reserve University

\section{Research Article}

Keywords: Faculty development, Promotion and retention, Course evaluation, Faculty satisfaction

Posted Date: June 2nd, 2021

DOI: https://doi.org/10.21203/rs.3.rs-544208/v1

License: (c) (i) This work is licensed under a Creative Commons Attribution 4.0 International License.

Read Full License 


\section{Abstract}

\section{Background}

Academic medical centers invest considerably in faculty development efforts to support the career success and promotion of their faculty and to minimize faculty attrition. This study evaluated the impact of a faculty development program called the Leadership in Academic Medicine Program (LAMP) on participants' (1) self-ratings of efficacy, (2) promotion in academic rank, and (3) institutional retention.

\section{Method}

Participants from the 2013-2020 LAMP cohorts were surveyed pre and post program to assess their level of agreement with statements that spanned domains of self-awareness, self-efficacy, satisfaction with work and work environment. Pre and post responses were compared using McNemar's tests. Changes in scores across gender were compared using Wilcoxon Rank Sum/Mann-Whitney tests.

LAMP participants were matched to nonparticipant controls by gender, rank, department, and time of hire to compare promotions in academic rank and departures from the organization. Kaplan Meier curves and Cox proportional hazards models were used to examine differences.

\section{Results}

There were significant improvements in almost all self-ratings on program surveys $(p<0.05)$. Greatest improvements were seen in "understand the promotions process" (36\% vs. $94 \%)$, "comfortable negotiating" ( $35 \%$ vs. $74 \%$ ), and "time management" (55\% vs. $92 \%$ ). There were no statistically significant differences in improvements by gender, however women faculty rated themselves lower on all preprogram items than men.

There was significant difference found in time-to-next promotion $(p=0.003)$ between LAMP participants and controls. Kaplan-Meier analysis demonstrated that LAMP faculty achieved next promotion more often and faster than controls. Cox-proportional-hazards analyses found that LAMP faculty were $61 \%$ more likely to get promoted than controls (hazard ratio $[\mathrm{HR}] 1.61,95 \%$ confidence interval $[\mathrm{Cl}] 1.16-2.23, \mathrm{p}$ value $=0.004)$.

There was significant difference found in time-to-departure $(p<0.0001)$ with LAMP faculty retained more often and for longer periods. LAMP faculty were $77 \%$ less likely to leave compared to controls (HR 0.23 , $95 \% \mathrm{Cl}$ 0.16-0.34, $\mathrm{p}<0.0001)$.

\section{Conclusions}

LAMP is an effective faculty development program as measured subjectively by participant self-ratings and objectively through comparative improvements in academic promotions and institutional retention. 


\section{Background}

The faculty are the lifeblood of academic medical centers and enabling their success is central to its mission. Multiple factors contribute to a faculty member's sense of career success, but self-efficacy and work enjoyment are fundamental. Faculty retention and academic promotions are additional measurements of academic success for organizations as well as for individuals. But achievement of faculty satisfaction, promotions and retention are not without challenges.

Only two thirds of medical faculty indicate satisfaction with their current careers, ${ }^{1,2,3}$ and almost one third consider leaving their medical college within the next 1-2 years. ${ }^{2,4}$ Faculty attrition, estimated at a rate of $5-8 \%$ annually, ${ }^{5,6,7}$ is often indicative of discontent and even burnout, which has negative effects on morale. ${ }^{1}$ For organizations faculty turnover is also costly, estimated at $\$ 400 K^{8}$ or twice the annual salary 5 of the departed faculty member. Faculty attrition is disproportionately high in several faculty groups including early career, non-white, female and clinical faculty $6,9,10,11$ which is problematic at a time when we need to diversify our workforce.

Academic promotion is also a struggle for many faculty and dissatisfaction with the clarity and reasonableness of promotions criteria is high. 2,12,13 Only one third of faculty achieve promotions from the assistant to the associate rank within 10 years and women, non-white and clinical faculty are less likely to accomplish this. ${ }^{14,15}$ For women, a persistent gap exists in the advancement at all ranks when compared to men. ${ }^{16,17,18}$

Faculty development programming is one method for addressing faculty satisfaction, ${ }^{19}$ effectiveness, ${ }^{20,21,22}$ academic promotions ${ }^{23,24}$ and retention, ${ }^{25}$ including amongst women faculty. ${ }^{26}$ However, prior studies do not distinguish curricular programs from funding efforts ${ }^{24}$ and combine promotion in academic rank with promotion in leadership roles, ${ }^{23}$ making it difficult to isolate the specific impacts of these interventions. Furthermore, retention outcomes are modest with $10-20 \%$ improvements even in nationally acclaimed programs, ${ }^{25,26}$ and are of unclear reproducibility in other academic medical centers.

\section{Method}

\section{Design and setting:}

At Weill Cornell Medicine, the Leadership in Academic Medicine Program (LAMP) is a 10-month long faculty development offering designed for early career faculty who serve or plan to serve in leadership roles. It consists of monthly afternoon sessions that cover the fundamentals of self-awareness, selfmanagement, career planning and leadership. In addition to seminars led by topic experts, LAMP also includes small groups with senior mentors as well as a capstone project. Adapted from a similar program at Indiana University School of Medicine, LAMP was piloted at Weill Cornell in 2012 in one Department 
and became a college wide offering in 2013. Program participants are nominated by their Department Chair and are competitively selected; $25-40$ faculty have enrolled annually.

The purpose of this study was to evaluate several outcomes of the 2013-2020 LAMP cohorts. First, we compared self-ratings of LAMP participants on self-awareness, self-efficacy, satisfaction with work and work environment before and after the program. Additionally, we evaluated differences in pre and post program self-ratings across gender. Second, we compared promotion in academic rank of LAMP participants compared to matched controls. Finally, we calculated retention of LAMP participants at Weill Cornell Medicine against matched controls. The study protocol was approved by the Weill Cornell Institutional Review Board.

\section{Study Sample:}

Between 2013 and 2020, a total of 242 faculty enrolled in LAMP (Figure 1). Of those, ten faculty were excluded due to missing data and/or because they did not complete the program. An additional 232 faculty who had not participated in LAMP were assembled to serve as controls for a total study sample of 464 faculty.

\section{Program surveys:}

LAMP participants were asked to complete a pre-program and post-program survey to identify career goals, programmatic needs, and existing skillset. One component of the survey asks the participants to rate (4-point Likert scale) their level of agreement with 14 statements on self-awareness, self-efficacy, satisfaction with work and work environment (Table 2). The survey was a stated expectation of program participation, administered online and at least two reminders were sent to encourage survey completion.

\section{Matched Controls:}

To assess ascension in academic rank and institutional retention, we assembled a cohort of 464 faculty, 232 LAMP graduates and 232 control faculty. To adjust for confounding effects of gender and environment, we matched each LAMP participant with 1 randomly selected nonparticipant from the institutional faculty database, a repository of all hired faculty. We matched for the following characteristics: (1) gender, (2) initial academic rank, (3) department and (4) hire year. When exact matches were not available, we allowed substitutions for closely related departments and collapsed year of hire into three-year increments. The following departmental combinations were made: (a) Biochemistry with Cell and Developmental Biology; (b) Neuroscience with Brain and Mind Research; (c) Radiation Oncology with Radiology; (d) Microbiology/Immunology and Genetic Medicine were combined with Medicine; and (e) Cardiothoracic surgery, Head and Neck surgery, Neurosurgery and Urology were combined to form Specialty Surgery. This grouping resulted in matches from 18 department groups, down from 26 initial departments. Finally, 6 LAMP participants required individualized assignments: 3 from Rehabilitative Medicine who were matched with controls from Medicine, 2 from Library matched to Oncology and Bioinformatics, and 1 from Surgery matched with Specialty surgery. 


\section{Data Analysis:}

To compare pre and post responses to the survey, McNemar's tests were done on dichotomized responses (Strongly Agree/Agree versus Strongly Disagree/Disagree). To evaluate differences in responses (original 4-point scale) from pre to post program by gender, we performed Wilcoxon Rank Sum/Mann-Whitney tests.

We constructed Kaplan- Meier curves from time of hire to promotion to next academic rank and to institutional departure for LAMP faculty and matched controls. For the analysis on time to next promotion, individuals were censored at the time of departure or at the end of the study, December $31^{\text {st }}$, 2020. For the analysis on time to departure, individuals were censored at the study end date if still employed. Group differences were assessed with log-rank tests. Cox-proportional-hazards models were used to measure the effect of LAMP participation on time to promotion and departure.

Recognizing that promotion from Assistant to Associate professor rank is different from promotion from Instructor to Assistant professor rank, we performed Kaplan-Meier analysis for the subgroup of Assistant Professors.

Analyses were conducted using Stata 15.1 (StataCorp LLC, College Station, TX) and results were considered significant at $p<0.05$.

\section{Results}

Demographic characteristics of LAMP faculty and matched controls can be found in Table 1. More women faculty (63.8\%) participated in LAMP than men (36.2\%). The majority $(62.1 \%)$ were hired at the rank of Assistant professor with the remaining (37.5\%) entering at the Instructor level; there was 1 Associate archivist accepted into the program as she was still early career. Caucasian race described $53.0 \%$ of the LAMP and the control cohort but more LAMP faculty self-identified as ethnic minority, Asian (31.0\%), Black (4.3\%) and Hispanic (4.3\%), than did controls $(24.1 \%, 3.0 \%$ and $1.7 \%$ respectively). LAMP faculty were drawn in largest numbers from the Departments of Medicine, Pediatrics and Anesthesiology. Participants were followed for an average of 5.3 years.

224 participants ( $96.6 \%$ response rate) completed both pre and post program surveys. Table 2 shows the changes in the \% agreement on 14 statements assessing self-awareness, self-efficacy, satisfaction with work and work environment. There were significant improvements in 11 of 14 items $(p<0.05)$. Greatest improvements were seen in "understand the promotions process" (36\% pre-program vs. $94 \%$ postprogram), "comfortable negotiating" ( $35 \%$ vs. $74 \%$ ), and "strategy for managing time" ( $55 \%$ vs. $92 \%$ ). Female faculty rated themselves lower on all items pre-program. The improvements in \% agreement was greatest amongst women compared to men in four items: "understand the promotions process", "comfortable negotiating", "confident in ability to present work" and "have skills to navigate success". No statistically significant differences were measured, however, between the improvements in the 4-point scores of women versus men. 
$163(35 \%)$ of the study subjects achieved promotion to the next academic rank within the study period, $108(46.6 \%)$ of the LAMP faculty and $55(23.7 \%)$ of the non-LAMP faculty. Cox-proportional-hazards analyses indicate that LAMP faculty were $61 \%$ more likely than controls to get promoted $(\mathrm{HR} 1.61,95 \% \mathrm{Cl}$ $1.16-2.23, p=0.004)$.

There was a significant difference found in time-to-next promotion $(p=0.003)$ between LAMP participants and controls. Kaplan-Meier curves reveal that LAMP faculty got to their next promotion more often and faster than controls (Fig. 2A). In the Assistant professor subgroup, there was a trend $(p=0.088)$ toward faster time to promotion to the Associate rank for LAMP participants (Fig. 2B).

There was a significant difference found in time-to-departure $(\mathrm{p}<0.0001)$ with LAMP faculty retained more often and for longer periods than controls (Fig. 3). Overall, $139(30 \%)$ of the subjects left Weill Cornell Medicine, 105 (45.3\%) of the non-LAMP controls and 34 (14.7\%) of the LAMP faculty. Faculty who participated in LAMP were $77 \%$ less likely to leave compared to controls (HR $0.23,95 \% \mathrm{Cl} 0.16-0.34, \mathrm{p}<$ 0.0001).

\section{Discussion}

Most faculty development programs evaluate for effect by surveying participant satisfaction. ${ }^{27}$ Few assess for higher levels of impact as defined by the Kirkpatrick model for educational assessment: learning, behavior and results. ${ }^{28}$ This study evaluated LAMP for subjective changes in participant ratings as well as objective differences in academic promotion and institutional retention.

We found significant improvements in LAMP graduates on all domains of self-awareness, self-efficacy, and satisfaction with work and work environment. Only 3 out of 14 survey items did not increase significantly as these baseline scores started high, with little to no room for improvement. Items where participants rated lowest at the start of the program (promotions, negotiation, and time management) saw the greatest improvements.

While the differences in the score improvements did not significantly differ by gender, it is noteworthy that women self-rated all pre-program items lower than men. Coupled with a higher enrollment of women into LAMP (64\%), this suggests that women may desire faculty development more than men and programs directed at women faculty might be needed.

We matched each LAMP participant to a faculty individual of the same gender, hired close to the same year, in the same or similar academic department, and at the same academic rank because these factors contribute to the career trajectory, promotions, and attrition of early career faculty. We achieved good specificity with our matching with preservation of over 18 different departments, although year of hire had to be expanded to a three-year increment.

Comparison to controls revealed that LAMP participants were $61 \%$ more likely to achieve promotion to the next academic rank and in a faster duration of time. We chose promotion to the next academic rank, 
instead of achievement of a specific rank, as our primary outcome because LAMP is designed to support early career faculty, both Instructors and Assistant professors, during a period of career vulnerability ${ }^{9}$. However, in acknowledgement of the fact that ascension to Associate professorship is generally considered more challenging than ascension to Assistant professorship, we performed Kaplan-Meier analysis on the Assistant professors. Subgroup analysis trended towards but did not reach statistical significance possibly due to the smaller number of these faculty or to the longer duration of time needed to get to Associate level.

Our study's most significant finding was institutional retention; LAMP faculty were retained more often and for longer periods compared to controls. Only $14.7 \%$ of the LAMP faculty left Weill Cornell Medicine during the study period compared to $45.3 \%$ of faculty who did not participate in LAMP. National data cite an annual faculty attrition rate of approximately $7 \% 5,6,7$ which corresponds to the departures in the control cohort. LAMP faculty were $77 \%$ less likely to leave, a remarkable return on investment from an organizational perspective. ${ }^{29}$ The greater representation of ethnic minority faculty in the LAMP group is also encouraging, although we cannot draw direct comparisons in retention since we did not match by race and ethnicity. Faculty retention is not an explicit goal of LAMP; the program encourages activities and roles that advance the participant's career even if outside of their home institution.

Finally, LAMP has the potential to impact not just those enrolled in the program, but others in their local environment, since faculty development has been shown to produce widespread learning. ${ }^{30}$

There were several limitations to our study. The dichotomization of survey responses from a 4-point Likert scale might have limited our ability to differentiate degrees of change within items. Promotions in rank is also an incomplete measure of career advancement and we would have liked to have assessed for additional achievements, including grants, awards, publications, and leadership roles. A major limitation of this study was the various confounders that influenced retention and promotion that we could not control for. All faculty accepted into LAMP are nominated by their Chair; this endorsement likely influences retention. Faculty who seek leadership or professional training are inherently interested in advancement and might seek additional training. We did not explore if or how often subjects sought faculty development training outside of LAMP, and one might assume controls are less likely to participate than LAMP faculty. While we cannot conclude causality from LAMP and our outcomes, the strong association affirms that LAMP contributes to the improvement, promotion, and retention of its faculty.

\section{Declarations}

Ethics and Consent: The surveys for the LAMP program were collected for internal programmatic feedback. The Institutional Review Board at Weill Cornell Medicine approved the study protocol (\#2001021388) to analyze the survey data retrospectively, waiving the requirement of informed consent. No biomedical, clinical or biometric data were obtained and all methods were carried out in accordance with relevant guidelines and regulations. 
Consent for publication: Not applicable

Availability of data and materials: The datasets generated and/or analyzed during the current study are not publicly available due to the local and proprietary nature of the data and because the data contained protected health information $(\mathrm{PHI})$ which is protected under HIPAA regulation, but are available from the corresponding author on reasonable request.

Competing interests: The authors declare that they have no competing interests.

Funding. The study was supported by the Office of Faculty Development, Weill Cornell Medicine.

Author's contributions: JT conceived and designed the study, acquired and interpreted the data, drafted and wrote the manuscript. MN contributed to the design of the study, acquired, analyzed and interpreted the data and edited the manuscript. MR contributed to the design of the study, analyzed and interpreted the data and edited the manuscript. LL conceived and designed the study, interpreted the data and edited the manuscript. All authors read and approved the final manuscript.

Acknowledgments: The authors thank Ms. Carolyn Hill, program manager of LAMP, the speakers and senior mentors who gave generously of their time and expertise to LAMP and especially the LAMP participants for sharing in and shaping the program. They also wish to thank Dr. Lisa Kern for her mentorship on the manuscript, Ms. Heather Olsen and Dean Jane Salmon for their assistance with the faculty database and Deans Katherine Hajjar and Augustine Choi for their support of the program.

\section{References}

1. Shanafelt T, West C, Sinsky $C$ et al. Changes in burnout and satisfaction with work-life integration in physicians and the general US working population between 2011 and 2017. Mayo Clin Proc 2019; 94 (9): 1681-94

2. Dandar V, Field J, Garrison G. Promising practices for promoting faculty engagement and retention at U.S. medical schools 2017. AAMC: 1-60

3. Simpkin A, Chang Y, Yu L, Campbell E, Armstrong K, Walensky R. Assessment of job satisfaction and feeling valued in academic medicine. JAMA Intern Med. 2019; 179 (7): 992-4

4. Pololi L, Krupat E, Civian J, Ash A, Brennan R. Why are a quarter of faculty considering leaving academic medicine? A study of their perceptions of institutional culture and intention to leave in 26 representative medical schools. Acad Med 2012; 87:859-69

5. Shanafelt T, Goh J, Sinsky C. The business case for investing in physician well-being. JAMA Int Med 2017; 177 (2): 1826-32

6. Corrice A, Fox S, Bunton S. Retention of full-time clinical MD faculty at US medical schools. AAMC Analysis in Brief 2011: 11 (2)

7. Alexander $\mathrm{H}$, Lang J. The long-term retention and attrition of US medical school faculty. AAMC Analysis in Brief 2008: 8 (4) 
8. Schloss E, Flanagan D, Culler C, Wright A. Come hidden costs of faculty turnover in clinical departments in one academic medical center. Acad Med 2009; 84(1):32-6

9. Liu C, Morrison E. US medical school full-time faculty attrition. AAMC Analysis in Brief $2014 ; 14$ (2)

10. Yamagata H. Trends in faculty attrition at US medical schools, 1980-1999. AAMC Analysis in Brief 2002; 2 (2)

11. Brod H, Lemeshow S, Binkley P. Determinants of faculty departure in an academic medical center: a time to event analysis. The Am J of Med 2017; 130 (4): 488-93

12. Jeffe D, Yan Y, Andriole D. Competing risks analysis of promotion and attrition in academic medicine: a national study of US medical school graduates. Acad Med 2019; 94 (2): 227-36.

13. Bunton S, Corrice A. Perceptions of the promotions process: an analysis of US medical school faculty. AAMC Analysis in Brief 2011; 11 (5)

14. Liu C, Alexander H. Promotion rates for first time assistant and associate professors appointed from 1967-1997. AAMC Analysis in Brief 2010; 9 (7)

15. Speck R, Sammel M, Troxel A et al. Factors impacting the departure rates of female and male junior medical school faculty: evidence from a longitudinal analysis. J of Women's Health 2012; 21 (10): 1059-65

16. Lautenberger D, Dander V. The state of women in academic medicine 2018-2019; exploring pathways to equity. AAMC 2020: 1-49

17. Richter $\mathrm{K}$, Clark L, Wick $\mathrm{J}$ et al. Women physicians and promotion in academic medicine. $\mathrm{N}$ Eng $\mathrm{J}$ Med 2020; 383: 2148-57

18. Carr P, Raj A, Kaplan S, Terrin N, Breeze J, Freund K. Gender differences in academic medicine: retention, rank and leadership comparisons from a national faculty survey. Acad Med 2018; 93 (11): 1694-9

19. Steinert Y, Mann K, Anderson B et al. A systematic review of faculty development initiatives designed to enhance teaching effectiveness: A 10-year update: BEME Guide No. 40. Medical Teacher 2016; 38 (8): 769-86

20. Hackworth J, Steel S, Cooksey E, DePalma M, Kahn J. Faculty member's self-awareness, leadership confidence, and leadership skills improve after an evidence-based leadership training program. J of Pediatrics 2018; 199: 4-6

21. Baldwin C, Gusic M, Chandran L. The impact of a national faculty development program embedded within an academic professional organization. Acad Med 2017; 92 (8): 1105-13

22. Bilal, Guraya S, Chen S. The impact and effectiveness of faculty development program in fostering the faculty's knowledge, skills and professional competence: a systematic review and meta-analysis. Saudi J of Bio Sci 2019; 29: 688-97

23. Haftel H, Swan R, Anderson M et al. Fostering the career development of future educational leaders: the success of the association of pediatric program director's leadership in educational academic development program. J of Pediatrics 2018; 194:5-6 
24. Connelly $M$, Sullivan $A$, Chinchilla $M$ et al. The impact of a junior faculty fellowship award on academic advancement and retention. Acad Med 2017; 92 (8): 1160-7

25. Ries A, Wingard D, Gamst A, Larsen C, Farrell E, Reznik V. Measuring faculty retention and success in academic medicine. Acad Med 2012; 87 (8): 1046-51

26. Chang $S$, Morahan $P$, Magrane $D$ et al. Retaining faculty in academic medicine: The impact of career development programs for women. J of Women's Health 2016; 25 (7): 687-96.

27. Lucas R, Goldman E, Scott A, Dander V. Leadership development programs at academic health centers: results from a national survey. Acad Med 2018; 93 (2): 229-36.

28. Kirkpatrick D. Evaluating training programs: the four levels. San Francisco: Berrett-Koehler 1994

29. Topor D, Roberts D. Faculty development programming at academic medical centers: identifying financial benefits and value. Med Sci Educ 2016; 26 (3): 417-9

30. Plack M, Goldman E, Wesner M, Manikoth N, Haywood Y. How learning transfers; a study of how graduates of a faculty education fellowship influenced the behaviors and practices of their peers and organization. Acad Med 2015; 90 (3): 372-8

\section{Tables}


Table 1

Subject Characteristics: Leadership in Academic Medicine Program (LAMP) 2013-2020

\begin{tabular}{|c|c|c|c|c|}
\hline \multirow{2}{*}{$\begin{array}{l}\text { Total sample }(\mathrm{N}=464) \\
\text { Gender }\end{array}$} & \multicolumn{2}{|c|}{ LAMP faculty $N=232$} & \multicolumn{2}{|c|}{ Controls $\mathrm{N}=232$} \\
\hline & & & & \\
\hline Female & 148 & $63.8 \%$ & 148 & $63.8 \%$ \\
\hline Male & 84 & $36.2 \%$ & 84 & $36.2 \%$ \\
\hline \multicolumn{5}{|l|}{ Initial Title } \\
\hline Instructor & 87 & $37.5 \%$ & 87 & $37.5 \%$ \\
\hline Assistant & 144 & $62.1 \%$ & 144 & $62.1 \%$ \\
\hline Associate & 1 & $0.4 \%$ & 1 & $0.4 \%$ \\
\hline \multicolumn{5}{|l|}{ Race/Ethnicity } \\
\hline Caucasian & 123 & $53.0 \%$ & 122 & $52.6 \%$ \\
\hline Asian & 74 & $31.0 \%$ & 56 & $24.1 \%$ \\
\hline Black & 10 & $4.3 \%$ & 7 & $3.0 \%$ \\
\hline Hispanic & 10 & $4.3 \%$ & 4 & $1.7 \%$ \\
\hline Other & 4 & $1.7 \%$ & 4 & $1.7 \%$ \\
\hline No response & 11 & $4.7 \%$ & 39 & $16.8 \%$ \\
\hline \multicolumn{5}{|l|}{ Department } \\
\hline Medicine & 85 & $36.6 \%$ & 86 & $37.1 \%$ \\
\hline Pediatrics & 26 & $11.2 \%$ & 26 & $11.2 \%$ \\
\hline Anesthesiology & 21 & $9.1 \%$ & 21 & $9.1 \%$ \\
\hline Pathology/Laboratory & 15 & $6.5 \%$ & 15 & $6.5 \%$ \\
\hline Psychiatry & 13 & $5.6 \%$ & 13 & $5.6 \%$ \\
\hline Surgery & 9 & $3.9 \%$ & 8 & $3.4 \%$ \\
\hline Obstetrics/Gynecology & 7 & $3.0 \%$ & 7 & $3.0 \%$ \\
\hline Radiology & 7 & $3.0 \%$ & 10 & $4.3 \%$ \\
\hline Neurology & 6 & $2.6 \%$ & 6 & $2.6 \%$ \\
\hline Radiation Oncology & 6 & $2.6 \%$ & 3 & $1.3 \%$ \\
\hline
\end{tabular}

LAMP faculty were matched by gender, academic rank, department, and time period of hire. They were not matched by race/ethnicity. 


\begin{tabular}{|lllll|}
\hline Total sample $\mathbf{N}=\mathbf{4 6 4})$ & \multicolumn{2}{c}{ LAMP faculty $\mathbf{N}=\mathbf{2 3 2}$} & \multicolumn{2}{c|}{ Controls $\mathbf{N}=\mathbf{2 3 2}$} \\
\hline Otolaryngology & 5 & $2.2 \%$ & 4 & $1.7 \%$ \\
\hline Rehabilitative Medicine & 5 & $2.2 \%$ & 2 & $0.9 \%$ \\
\hline Library & 4 & $1.7 \%$ & 2 & $0.9 \%$ \\
\hline Ophthalmology & 4 & $1.7 \%$ & 4 & $1.7 \%$ \\
\hline Brain and Mind Research & 3 & $1.3 \%$ & 2 & $0.9 \%$ \\
\hline Neurosurgery & 3 & $1.3 \%$ & 3 & $1.3 \%$ \\
\hline Urology & 3 & $1.3 \%$ & 2 & $0.9 \%$ \\
\hline Emergency Medicine & 2 & $0.9 \%$ & 2 & $0.9 \%$ \\
\hline Genetic Medicine & 2 & $0.9 \%$ & 4 & $1.7 \%$ \\
\hline Population Health Sciences & 2 & $0.9 \%$ & 2 & $0.9 \%$ \\
\hline Biochemistry & 1 & $0.4 \%$ & 1 & $0.4 \%$ \\
\hline Dermatology & 1 & $0.4 \%$ & 1 & $0.4 \%$ \\
\hline Microbiology and Immunology & 1 & $0.4 \%$ & 3 & $1.3 \%$ \\
\hline Neuroscience & 1 & $0.4 \%$ & 2 & $0.9 \%$ \\
\hline LAMP faculty were matched by gender, academic rank, department, and time period of hire. They were \\
not matched by race/ethnicity.
\end{tabular}

Due to technical limitations, table 2 is only available as a download in the Supplemental Files section

\section{Figures}




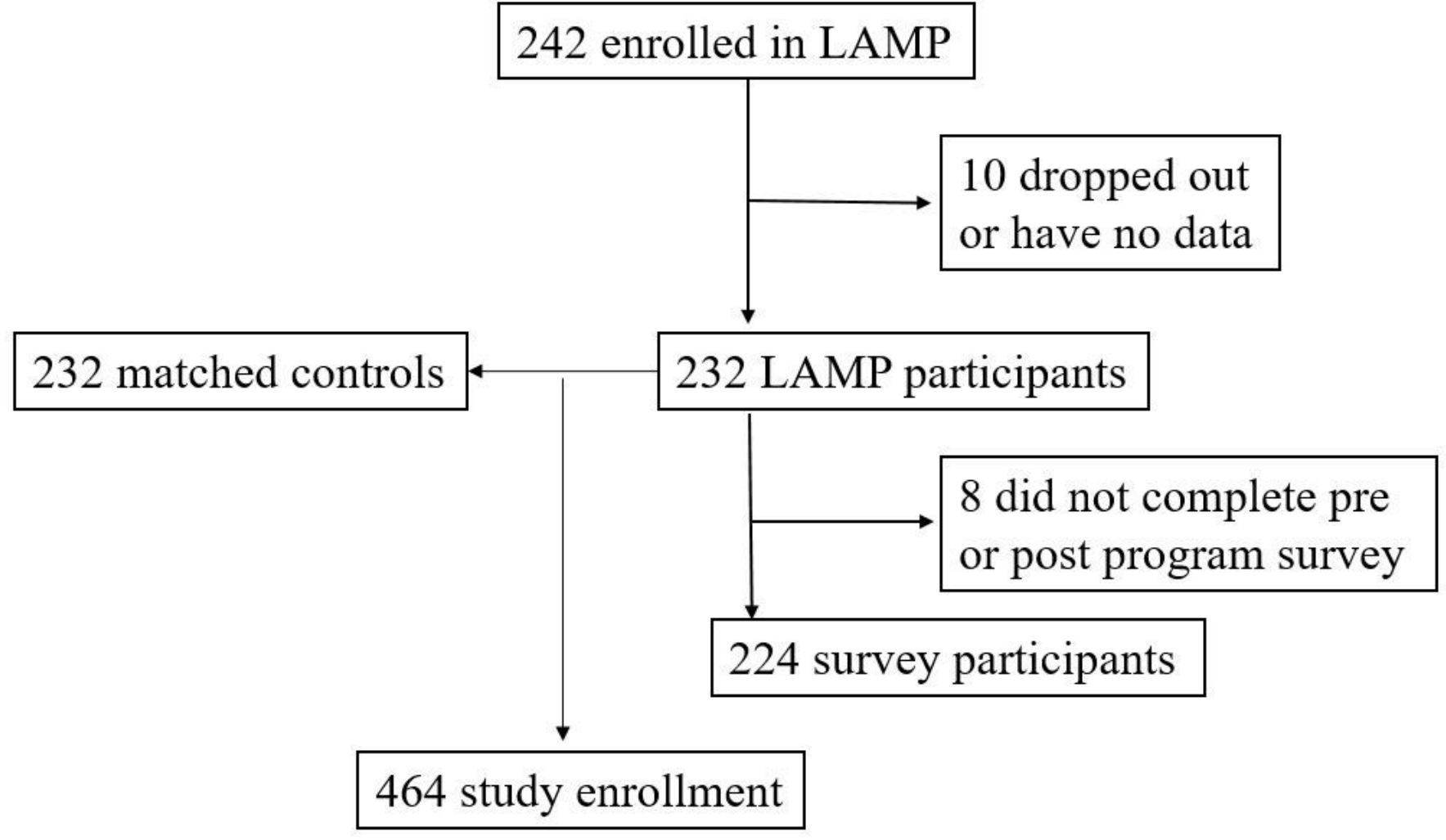

Figure 1

Study enrollment in the Leadership in Academic Medicine Program (LAMP) from 2013-2020

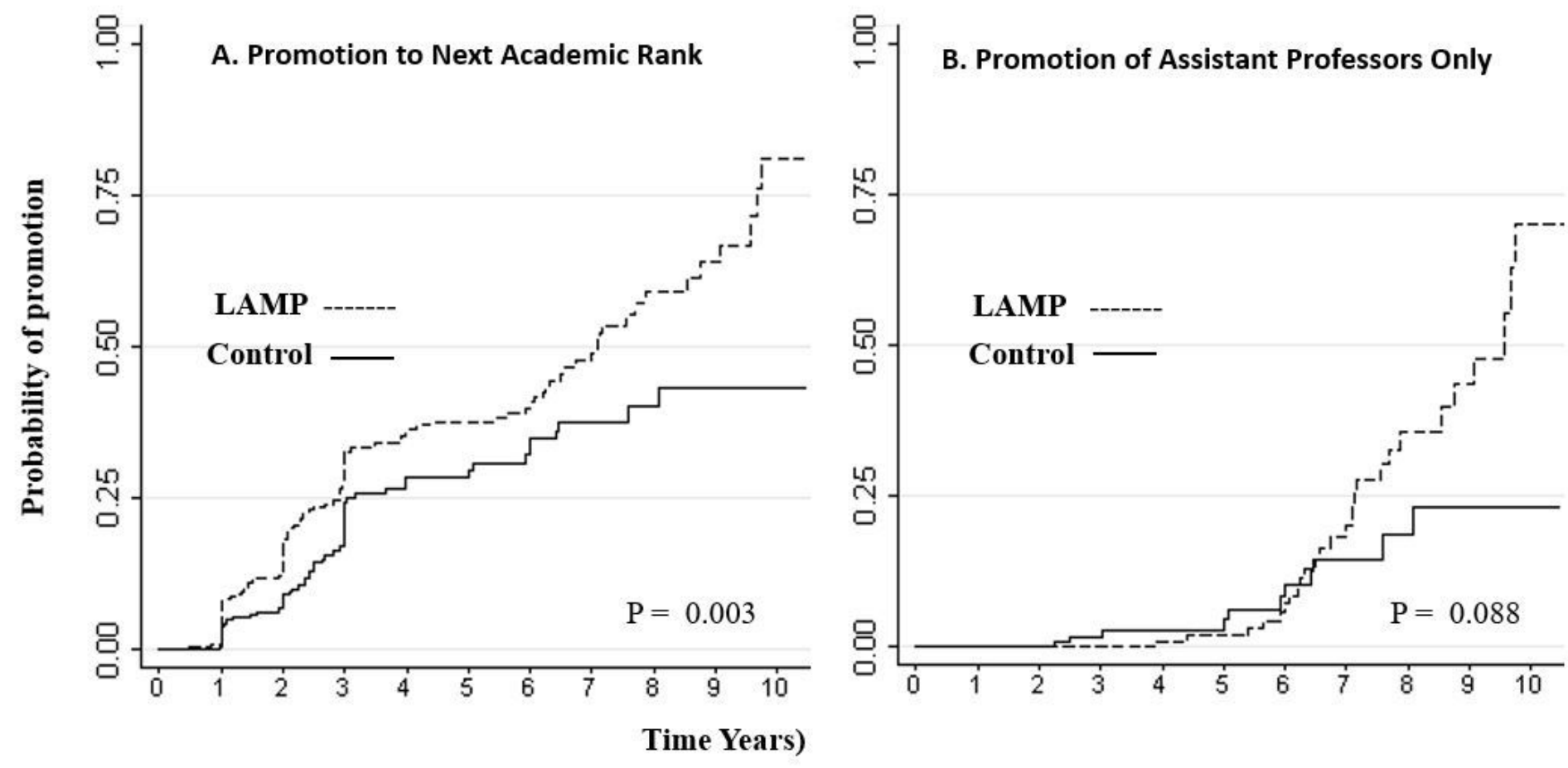

Figure 2 
Kaplan-Meier analysis for promotion in academic rank for LAMP faculty and matched controls from year of hire to 2020. A. Promotion to next academic rank for all study subjects: Instructor to Assistant and Assistant to Associate rank B. Promotions for Assistant professors only: Assistant to Associate rank

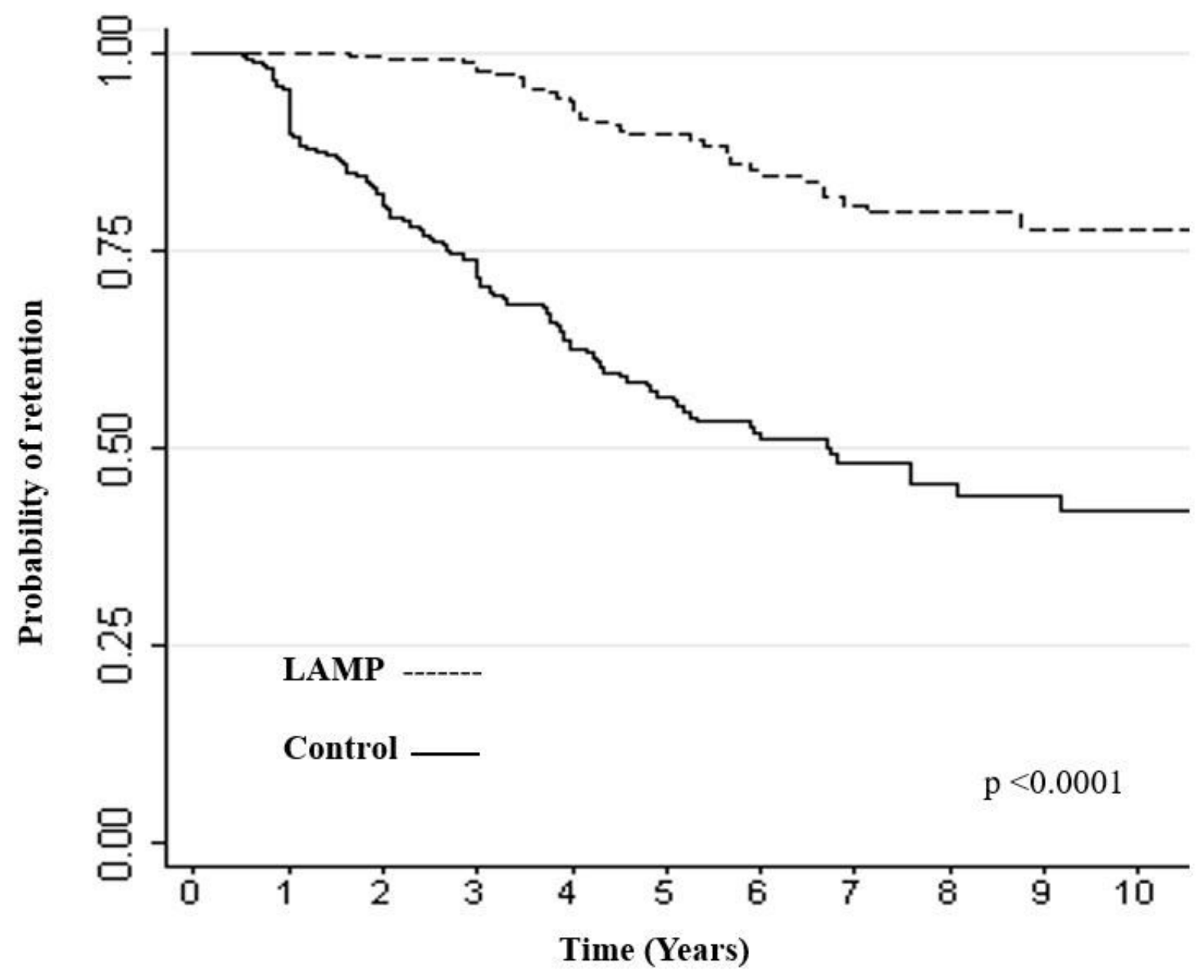

Figure 3

Kaplan-Meier analysis for the institutional retention of LAMP faculty and matched controls from year of hire through 2020. $P<0.0001$ by log-rank test.

\section{Supplementary Files}

This is a list of supplementary files associated with this preprint. Click to download.

- Table2.pptx 